\title{
Transperitoneal laparoscopic adrenalectomy: a review and single-center experience
}

\author{
Kamol Panumatrassamee, Manint Usawachintachit, Supoj Ratchanon, Apirak Santi-ngamkun \\ Division of Urology, Department of Surgery, Faculty of Medicine, Chulalongkorn University, Bangkok \\ 10330, Thailand
}

\begin{abstract}
Background: Laparoscopic adrenalectomy (LA) is the preferred approach to adrenal gland tumors.
Objective: To evaluate the perioperative and functional outcomes of transperitoneal LA.

Methods: We retrospectively reviewed data of all consecutive patients who underwent transperitoneal LA at our institution between April 2000 and December 2011.

Results: Two hundred two patients with mean age of 46 years were included in the study. There were 109 left, 89 right, and 4 bilateral tumors. Preoperative diagnosis included 126 aldosterone-producing adenomas (APA), 33 patients with Cushing syndrome, 19 pheochromocytomas, 20 nonfunctional tumors, and 4 adrenal metastases. Mean tumor size was $3.3 \mathrm{~cm}$ (range 0.4-22 cm). Mean operative time was $109 \mathrm{~min}$ and estimated blood loss $90 \mathrm{~mL}$ for unilateral adrenalectomy; and $195 \mathrm{~min}$ and $300 \mathrm{~mL}$ for bilateral adrenalectomy. Four patients with pheochromocytoma required transfusion and 2 required conversion to an open approach. Seven intraoperative complications and 18 30-day postoperative complications occurred. These included 5 major complications (Clavien-Dindo grade 3-5). Ninety-seven percent of APA patients had normal serum potassium levels after surgery. Among the patients with functioning endocrine tumors, complete resolution rate from secondary hypertension was significantly higher in the pheochromocytoma group compared with those with APA or Cushing syndrome ( $82 \%$ vs. $48 \%$ vs. $68 \%$ respectively, $p=0.02$ ). Mean follow up was 35.2 months.

Conclusion: LA is the treatment of choice for most adrenal gland tumors with excellent outcomes and low complication rates. There was a high chance for complete resolution of secondary hypertension after surgery for pheochromocytoma.
\end{abstract}

Keywords: Adrenalectomy, adrenal glands, laparoscopy, resolution of hypertension

It has been over 20 years since laparoscopic adrenalectomy (LA) was first described [1]. The surgical management of adrenal tumors by this minimally invasive approach has been commonly accepted worldwide. LA has become the operative procedure of choice for most adrenal tumors [2]. Many studies have reported the efficacy and safety of LA $[3,4]$. The small size of adrenal glands, located in the retroperitoneal space surrounded by major intraabdominal organs and great vessels, makes LA an ideal approach. The recognized benefits over an open approach include decreased blood loss, reduced postoperative pain, shortening of the recovery period, and better cosmetic results [5]. We performed the first LA at our institute in 2000. All the operations were performed using lateral transperitoneal approach.

Correspondence to: Apirak Santi-ngamkun, MD, Division of Urology, Department of Surgery, Faculty of Medicine, Chulalongkorn University, Bangkok 10330, Thailand. E-mail:apirak99@hotmail.com,kamol_uro@hotmail.com
Size of the tumor and the preoperative diagnosis were not a contraindication, but depended on the surgeon's decision. The aim of this study was to evaluate the perioperative and long-term functional outcomes, and the safety of our LA technique from 11 years of experience.

\section{Materials and methods Study population}

After the study protocol was approved by the Ethics Committee of our institution, we retrospectively reviewed medical records of all LA cases at our institution between April 2000 and December 2011. A total of 202 consecutive patients (57 male and 145 female) underwent LA performed by three surgeons. Unilateral LA was performed in 109 patients on the left side and 89 patients on the right side. Bilateral LAs were performed in four patients. In total, 206 adrenal glands were removed. Preoperative indications were 126 aldosterone-producing adenomas 
(APA), 33 patients with Cushing syndrome, 19 pheochromocytomas, 20 nonfunctioning tumors, and 4 adrenal metastases.

The medical records of all patients were systematically reviewed and the demographic data, operative techniques, perioperative parameters, functional outcomes, and long term follow-up results were analyzed.

\section{Preoperative preparation}

All patients were first evaluated by endocrinologists. The size of tumor and its extension were evaluated by computed tomography (CT) or magnetic resonance imaging (MRI).

In the APA patients, the morning plasma aldosterone concentration and the plasma renin activity were measured after correction of hypokalemia. Subsequently, a 2-litre normal saline loading test and an adrenal vein sampling test were performed to confirm diagnosis. In patients with a cortisol-secreting tumor (Cushing syndrome), we measured the plasma morning cortisol and the 24-h urine cortisol. Then, a high dose dexamethasone suppression test was performed. A hydrocortisone replacement dose was given to all patients at the day of surgery and was gradually reduced in the postoperative period.

In patients with pheochromocytoma, plasma and 24-h urine metanephrines/normetanephrines were measured. MRI was preferable to CT for tumor localization. Patient blood pressure was controlled with a titratable dose of nonselective $\alpha$-blocker at least two weeks before surgery, and $\beta$-blocker was added if tachycardia occurred. We suggested increased intake of fluid before surgery to increase intravascular volume. In patients with nonfunctioning tumors, indication for LA was a tumor larger than $5 \mathrm{~cm}$. Tumors with adjacent organ invasion were managed by an open approach.

\section{Surgical technique}

One gram of cephazolin was given as a prophylactic antibiotic. No bowel preparation was routinely used. After general anesthesia, a nasogastric (or orogastric) tube and urethral catheter were inserted. The patient was placed in a lateral decubitus position with side of the lesion up and the kidney was slightly elevated. The lower leg was flexed at 90 with the upper leg in a straight position and all the pressure points supported. Bilateral LA patients were repositioned after finishing the first side.
The first port (12 mm, camera) was inserted using the Hasson technique at the midclavicular line, $5 \mathrm{~cm}$ cephalad from the umbilicus. Pneumoperitoneum was created with carbon dioxide and maintained at the level between 12 to $15 \mathrm{mmHg}$. End-tidal $\mathrm{CO}_{2}$ was monitored during the operation. Two 5-mm working ports were inserted below the costal margin under direct vision with a triangular principle. In some patients, if more exposure was required, the fourth port for the retracting instrument was inserted at the subxiphoid region for the right side tumor and at the left midaxillary line below the costal margin for the left side tumor.

The line of Toldt was incised and colon was mobilized medially. The adrenal vein was firstly identified and controlled with surgical clips or LigaSure (Covidien, Manfield, MA, USA), depended on its size and surgeon's preferences. The adrenal gland was dissected from the surrounding attachments. First, the superior border was separated from the liver and spleen. Then, the medial border was separated from the inferior vena cava and aorta. Last, the inferior border was dissected from the upper pole of the kidney. The specimen was placed in a retrieval bag and removed through the camera port-site. A close suction drain was placed before incisions were closed.

\section{Data analysis}

We analyzed variables included age, gender, body mass index (BMI), tumor laterality, tumor size, operative time, estimated blood loss (EBL), intraoperative and postoperative complications, frequency of conversion to open surgery and need for transfusion, method to control adrenal vein, narcotics requirement, postoperative hospital stay, tumor histology, and functional outcomes.

Tumor size was recorded using the largest dimension from the pathology reports. Complications within 30 days after surgery were graded according to the standardized Clavien-Dindo classification [6]. We reported the postoperative narcotics requirement in morphine equivalents and postoperative hospital stay instead of total hospital stay because some patients in our country tend to stay in the hospital during the diagnostic period.

Analysis was performed with the JMP 9 software (SAS institute Inc, Cary, NC, USA). Continuous variables were reported as mean (standard deviation) and categorical variables were reported as a number (percentage). A Pearson Chi-square test was used to compare the data between groups. 


\section{Results}

Overall demographic data are summarized in Table 1. Mean age was 46 years (range, 11 to 77 years) and mean tumor size was $3.3 \mathrm{~cm}$ (range 0.4 to $22 \mathrm{~cm}$ ). Perioperative outcomes are reported in Table 2. Four patients with pheochromocytoma required blood transfusion and two of them, with large tumors ( 8 and $9 \mathrm{~cm}$ ), were converted to open surgery because of bleeding and extensive adhesion.

The mean postoperative narcotics requirement was $7 \mathrm{mg}$ morphine equivalent. Thirty-six patients (17.8\%) did not require narcotics for postoperative pain. Patients returned to a full oral diet at mean of 2.5 days postoperatively and the mean postoperative hospital stay was 4.5 days.

Intraoperative complications occurred in 7 patients (3.5\%) including 3 liver injuries and 1 splenic injury, which were managed with cautery hemostasis. One colonic injury, 1 duodenal serosal injury, and 1 diaphragmatic injury were repaired with laparoscopic suturing. All of these patients had an uneventful postoperative period. Eighteen (9\%) postoperative complications occurred in this study. There were 13 minor complications (Clavien-Dindo grade 1-2) including 5 infected wounds, 4 transfusions, 2 prolonged ileus, 1 reversible acute renal failure, and 1 subcutaneous emphysema. Major complications (Clavien grade 3-4) occurred in 5 patients; 1 patient with pheochromocytoma had right hemiparesis from cerebral infarction, but fully recovered after 2 weeks of rehabilitation (Clavien grade 4a); 1 patient had postoperative bleeding and required laparotomy
(Clavien grade 3b); 2 patients had incisional hernia at the camera port that were subsequently repaired with mesh (Clavien grade 3b); 1 patient had vocal cord swelling and required reintubation (Clavien grade $3 a$ ); no perioperative mortality occurred in any patient in this study.

Bilateral LAs were performed in 4 patients with hypercortisolism. All of them were female; 2 patients were diagnosed Cushing syndrome with failure after pituitary surgery and radiation, 2 patients had ectopic adrenocorticotrophic hormone dependent syndrome with bilateral adrenal hyperplasia, and we were unable to identify the primary tumor. All these 4 patients had an uneventful perioperative period and received lifelong hormone replacement therapy.

We used the LigaSure (Covidien, Manfield, MA, USA) to control of the adrenal vein in our most recent 119 patients (59\%) with a small size tumor (clipless LA). The decision was made based on the size of the vein (less than $5 \mathrm{~mm}$ ) and the surgeons' preferences. There were no adverse postoperative events using this technique.

The histopathology reports are summarized in Table 3. There were 6 (3\%) primary malignancies with the mean tumor size $6.4 \mathrm{~cm}(2.5-12 \mathrm{~cm})$ including 5 adrenocortical carcinoma (ACC), and 1 malignant pheochromocytoma. All surgical margins were normal. Among the ACC patients, hypercortisolism was the presenting symptom in 4 patients (80\%), and one patient presented with a nonfunctioning tumor. No port site tumor recurrence, local recurrence or metastasis occurred during the follow-up period.

Table 1. Demographic data

\begin{tabular}{ll}
\hline Variables & \\
\hline Number of patients & 202 \\
Sex, male/female, n(\%) & $57(28) / 145(72)$ \\
Tumor laterality, right/left/bilateral, n (\%) & $89(44) / 109(54) / 4(2)$ \\
Age, year, mean (SD)/range & $46(12.8) / 11-77$ \\
BMI, kg/m², mean (SD)/range & $24.9(4.6) / 15-40.2$ \\
Tumor size, cm, mean (SD)/range & $3.3(2.6) / 0.4-22$ \\
Preoperative diagnosis, n (\%) & $126(62.4)$ \\
$\quad$ Aldosterone producing adenoma & $33(16.3)$ \\
$\quad$ Cushing syndrome & $19(9.4)$ \\
$\quad$ Pheochromocytoma & $20(9.9)$ \\
$\quad$ Adrenal metastasis & $4(2)$ \\
\hline
\end{tabular}

SD = standard deviation 
Table 2. Perioperative outcomes

\begin{tabular}{ll}
\hline Variables & \\
\hline $\begin{array}{l}\text { Operative time, min, mean (SD)/range } \\
\quad \text { Unilateral adrenalectomy }\end{array}$ & $108(44) / 45-420$ \\
$\quad$ Bilateral adrenalectomy & $195(52) / 150-240$ \\
Estimated blood loss, mL, mean (SD)/range & \\
$\quad$ Unilateral adrenalectomy & $90(217) / 10-2500$ \\
$\quad$ Bilateral adrenalectomy & $300(187) / 50-500$ \\
Transfusion, n (\%) & $4(2)$ \\
Open conversion, n (\%) & $2(1)$ \\
Intraoperative complications, n (\%) & $7(3.5)$ \\
Postoperative analgesic requirement, mg of morphine equivalent, mean (SD)/range & $7(6.6) / 0-35$ \\
Postoperative hospital stay, day, mean (SD)/range & $4.5(3) / 2-30$ \\
Overall postoperative complications, n (\%) & $18(9)$ \\
$\quad$ Minor (Clavien-Dindo grade 1-2), n (\%) & $13(6.5)$ \\
$\quad$ Major (Clavien-Dindo grade 3-5), n (\%) & $5(2.5)$ \\
Follow up length, month, mean (SD)/range & $35.2(30) / 0.5-120$ \\
\hline
\end{tabular}

$\mathrm{SD}=$ standard deviation

Table 3. Histopathological reports

\begin{tabular}{cl}
\hline No. of patients & Pathology \\
\hline 126 & Cortical adenoma \\
31 & Nodular hyperplasia \\
12 & Pheochromocytoma \\
5 & Cortical hyperplasia, adrenocortical carcinoma, myelolipoma \\
3 & Ganglioneuroma, adrenal hemorrhage \\
2 & Adrenocortical oncocytic adenoma, paraganglioma, hemorrhagic cyst, adrenal inflammation \\
1 & Fibrocalcified nodule, mature cystic teratoma, metastatic colon cancer, malignant pheochromocytoma
\end{tabular}

\section{Functional outcomes}

All of the APA patients had hypokalemia before surgery. Ninety-seven percent of these patients had normal serum potassium levels after surgery. Only 4 patients (3\%) still had persistent hypokalemia and were treated with potassium-sparing diuretic.

Eighty percent of patients in this study had secondary hypertension before surgery, including $95 \%$ of the APA group, $76 \%$ of the patients with Cushing syndrome, and $90 \%$ of the pheochromocytoma group. After surgery, 53\% of these patients had normalization of blood pressure without requiring any antihypertensive medication. The incidence of complete resolution was significantly higher in the pheochromocytoma patients compared with the APA and the patients with Cushing syndrome ( $82 \%$ vs. $48 \%$ vs. $68 \%$ respectively, $P=0.02$ ). The mean follow up was 35.2 months.

\section{Discussion}

With an increasing availability of abdominal imaging technology, adrenal lesions requiring surgical removal have been more often identified [7]. Open adrenalectomy has been mostly replaced by LA for over a decade. Numerous studies have demonstrated the safety and efficacy of LA in different approaches $[4,8]$. At present, no clear advantage between the transperitoneal and retroperitoneal approaches has been shown [9]. Because of the large working space with familiar anatomical landmarks, we performed a transperitoneal approach in all patients.

In the early period, LA was used only in benign adrenal masses $<6 \mathrm{~cm}$ and $<100 \mathrm{~g}$ [10]. Recently, with growth of surgical experience and improvement of surgical instrumentation, indications of LA were expanded. Previous contraindications, including large tumor, obesity, previous abdominal surgery, malignancy, and pheochromocytoma have been discarded [11-15]. 
Tumor size correlates directly with the risk of malignancy and technical difficulty. These are two major factors of concern in LA for large tumors. The role of LA in adrenocortical carcinoma (ACC) is controversial. ACC is a deadly disease with high rate of recurrence and poor prognosis. Surgical removal with a negative margin is the most important part of treatment. Miller et al. [16] suggested an open approach in patients suspected of, or with known ACC. By contrast, Brix et al. [17] reported no significant difference between an open and laparoscopic approach for a localized ACC with a size $<10 \mathrm{~cm}$. Excellent preoperative imaging and intraoperative laparoscopic ultrasound are useful tools for evaluation of tumor extension and invasion before surgical resection. In our experience, 26 patients had tumors larger than $6 \mathrm{~cm}$. Although some patients in our study were suspected of having ACC before surgery, we decided to use a laparoscopic approach because tumors were well-encapsulated and there was no adjacent organ invasion. All patients had negative margins and no recurrence occurred during the follow up.

Management of pheochromocytoma is more complex. Key factors are blood pressure control, adequate intravascular volume replacement, and close monitoring during surgery [18]. Because of the excessive catecholamine release during tumor manipulation and risk of hypotension from the withdrawal effect after dividing the adrenal vein, patients with pheochromocytoma are always classified as being in a high risk group. Early control of the adrenal vein with minimal tumor manipulation is a benefit of well performed LA in pheochromocytoma [19]. There were 19 pheochromocytoma patients in our study. Two of these, with large tumors, required open conversion; however, both instances occurred during our first year of experience.

Bilateral simultaneous LA was performed as two LAs in a single patient during the same session. Operative time was prolonged by the second operation and repositioning of the patient. The major concerns are the postoperative care with lifelong hormone replacement and the need to adjust dose over time. Patients in this group tend to have more complications including poor wound healing, infection, and bleeding and thromboembolic events from the usually long hypercortisolism state before surgery [20].

Assalia and Gagner [21] reported a meta-analysis from 28 centers where 2550 LAs were performed between January 1990 and October 2003. The mean operative time was $156 \mathrm{~min}$, mean EBL was $104 \mathrm{~mL}$, the mean conversion rate was $3.6 \%$, and mean complication rate was $9.5 \%$. More recently, Wang et al. [22] reviewed the indications, surgical techniques and the outcomes of LA. Our results were comparable with both of these studies. Our mean postoperative hospital stay (4.5 day) was longer than in the other series. This is considered to be the result of free or lower hospital costs to Thai patients and the nature of Thai patients (often rural based and distant from a hospital), who wanted to stay in the hospital until they were fully recovered.

An excellent functional outcome is the primary goal of adrenalectomy for functioning adrenal tumors. In this study, $3 \%$ of the APA patients had persistent hypokalemia and high plasma aldosterone levels after surgery. We concluded that bilateral adrenal hyperplasia or idiopathic hyperaldosteronism was the cause of hyperaldosteronism in these patients. This situation might occur after an incorrect diagnosis, especially in patients with a small adenoma and where the adrenal vein sampling test could not be performed.

Secondary hypertension from a functioning adrenal mass has different mechanisms depending on the diagnosis [23]. A previous study reported predictive factors for resolution of hypertension in APA after surgery [24]. However, the underlying cause of persistent hypertension is still controversial. In our study, the rate of complete resolution from secondary hypertension was significantly higher in pheochromocytoma compared with APA and in patients with Cushing syndrome groups.

Clipless and sutureless techniques in LA have been described previously. Various instruments have been used to control the adrenal vessels [25, 26]. Advantages in reduction of the risk of adrenal vein avulsion and clip dislodgement showed these techniques to be preferable in most of the patients with a small tumor. In our experience, we always used the LigaSure to control the adrenal vessels in all of the APA patients as well as in the patients with Cushing syndrome. We concluded this technique is safe, effective, and can reduce the operative time without increased complications.

Recently, laparoendoscopic single-site surgery (LESS) and robotic-assisted surgery have been increasingly performed for adrenal surgery. LESS is a step forward of minimally invasive surgery to minimize the surgical scar. The transumbilical approach 
provides a better cosmetic result. However, the technique is more difficult in dissection and retraction because of the long distance between the umbilicus and the adrenal gland [27]. A robotic platform provides advantages over laparoscopy in some operations. However, no clear advantage of robotic adrenalectomy has been found at present [28]. Robotic adrenalectomy may be useful in the posterior retroperitoneal approach where the tumor is located more superiorly [29] and in the cortical-sparing adrenalectomy procedure [30].

Limitations of this study include that it is retrospective and descriptive. There was no comparison of the perioperative outcomes between the diagnoses. Nevertheless, the findings of this study demonstrate the safety and feasibility of our surgical technique in LA.

\section{Conclusions}

Laparoscopic adrenalectomy is the treatment of choice for various types of adrenal tumors. Excellent surgical outcomes with low complication rates have been demonstrated. The clipless technique is safe and feasible for most small tumors. Patients with pheochromocytoma have a high chance of complete resolution of secondary hypertension.

\section{Abbreviations}

ACC = adrenocortical carcinoma; APA = aldosterone producing adenoma; EBL = estimated blood loss; LA = laparoscopic adrenalectomy; LESS = laparoendoscopic single-site surgery

\section{Acknowledgments}

This study was presented in part at the 11th Asian Congress of Urology of the Urological Association of Asia, 22-26 August 2012, Pattaya, Thailand. The abstract was published in the International Journal of Urology, Volume 19, Issue Supplement s1, page 307, August 2012. None of the authors have any conflict of interest to declare.

\section{References}

1. Gagner M, Lacroix A, Bolte E. Laparoscopic adrenalectomy in Cushing's syndrome and pheochromocytoma. N Engl J Med. 1992; 327:1033.

2. Smith CD, Weber CJ, Amerson JR. Laparoscopic adrenalectomy: new gold standard. World J Surg. 1999; 23:389-96.

3. Terachi T, Matsuda T, Terai A, Ogawa O, Kakehi Y,
Kawakita M, et al. Transperitoneal laparoscopic adrenalectomy: experience in 100 patients. J Endourol. 1997; 11:361-5.

4. Kulis T, Knezevic N, Pekez M, Kastelan D, Grkovic M, Kastelan Z. Laparoscopic adrenalectomy: lessons learned from 306 cases. J Laparoendosc Adv Surg Tech A. 2012; 22:22-6.

5. Ramachandran MS, Reid JA, Dolan SJ, Farling PA, Russell CF. Laparoscopic adrenalectomy versus open adrenalectomy: results from a retrospective comparative study. Ulster Med J. 2006; 75:126-8.

6. Dindo D, Demartines N, Clavien PA. Classification of surgical complications: a new proposal with evaluation in a cohort of 6336 patients and results of a survey. Ann Surg. 2004; 240:205-13.

7. Taffel M, Haji-Momenian S, Nikolaidis P, Miller FH. Adrenal imaging: a comprehensive review. Radiol Clin North Am. 2012; 50:219-43.

8. Zhang X, Fu B, Lang B, Zhang J, Xu K, Li HZ, et al. Technique of anatomical retroperitoneoscopic adrenalectomy with report of 800 cases. J Urol. 2007; 177:1254-7.

9. Rubinstein M, Gill IS, Aron M, Kilciler M, Meraney AM, Finelli A, et al. Prospective, randomized comparison of transperitoneal versus retroperitoneal laparoscopic adrenalectomy. J Urol. 2005; 174:442-5.

10. MacGillivray DC, Whalen GF, Malchoff CD, Oppenheim DS, Shichman SJ. Laparoscopic resection of large adrenal tumors. Ann Surg Oncol. 2002; 9: 480-5.

11. Zografos GN, Farfaras A, Vasiliadis G, Pappa T, Aggeli C, Vasilatou E, et al. Laparoscopic resection of large adrenal tumors. JSLS. 2010; 14:364-8.

12. Fazeli-Matin S, Gill IS, Hsu TH, Sung GT, Novick AC. Laparoscopic renal and adrenal surgery in obese patients: comparison to open surgery. J Urol. 1999; 162:665-9.

13. Morris L, Ituarte P, Zarnegar R, Duh QY, Ahmed L, Lee J, et al. Laparoscopic adrenalectomy after prior abdominal surgery. World J Surg. 2008; 32:897-903.

14. Zografos GN, Vasiliadis G, Farfaras AN, Aggeli C, Digalakis M. Laparoscopic surgery for malignant adrenal tumors. JSLS. 2009; 13:196-202.

15. Toniato A, Boschin IM, Opocher G, Guolo A, Pelizzo M, Mantero F. Is the laparoscopic adrenalectomy for pheochromocytoma the best treatment? Surgery. 2007; 141:723-7.

16. Miller BS, Ammori JB, Gauger PG, Broome JT, Hammer GD, Doherty GM. Laparoscopic resection is inappropriate in patients with known or suspected 
adrenocortical carcinoma. World J Surg. 2010; 34: 1380-5.

17. Brix D, Allolio B, Fenske W, Agha A, Dralle H, Jurowich C, et al. Laparoscopic versus open adrenalectomy for adrenocortical carcinoma: surgical and oncologic outcome in 152 patients. Eur Urol. 2010; 58:609-15.

18. Bruynzeel H, Feelders RA, Groenland TH, van den Meiracker AH, van Eijck CH, Lange JF, et al. Risk factors for hemodynamic instability during surgery for pheochromocytoma. J Clin Endocrinol Metab. 2010; 95:678-85.

19. Janetschek G, Neumann HP. Laparoscopic surgery for pheochromocytoma. Urol Clin North Am. 2001; 28: 97-105.

20. Takata MC, Kebebew E, Clark OH, Duh QY. Laparoscopic bilateral adrenalectomy: results for 30 consecutive cases. Surg Endosc. 2008; 22:202-7.

21. Assalia A, Gagner M. Laparoscopic adrenalectomy. Br J Surg. 2004; 91:1259-74.

22. Wang DS, Terashi T. Laparoscopic adrenalectomy. Urol Clin North Am. 2008;3 5:351-63.

23. Sukor N. Endocrine hypertension - Current understanding and comprehensive management review. Eur J Intern Med. 2011; 22:433-40.

24. Zarnegar R, Young WF Jr, Lee J, Sweet MP, Kebebew E, Farley DR, et al. The aldosteronoma resolution score: predicting complete resolution of hypertension after adrenalectomy for aldosteronoma. Ann Surg. 2008; 247:511-8.

25. Misra MC, Aggarwal S, Guleria S, Seenu V, Bhalla AP. Clipless and sutureless laparoscopic surgery for adrenal and extra-adrenal tumors. JSLS. 2008; 12: 252-5.

26. Surgit O. Clipless and sutureless laparoscopic adrenalectomy carried out with the LigaSure device in 32 patients. Surg Laparosc Endosc Percutan Tech. 2010; 20:109-13.

27. Shi TP, Zhang X, Ma X, Li HZ, Zhu J, Wang BJ, et al. Laparoendoscopic single-site retroperitoneoscopic adrenalectomy: a matched-pair comparison with the gold standard. Surg Endosc. 2011; 25:2117-24.

28. Brunaud L, Bresler L, Ayav A, Zarnegar R, Raphoz AL, Levan T, et al. Robotic-assisted adrenalectomy: what advantages compared to lateral transperitoneal laparoscopic adrenalectomy? Am J Surg. 2008; 195: 433-8.

29. Berber E, Mitchell J, Milas M, Siperstein A. Robotic posterior retroperitoneal adrenalectomy: operative technique. Arch Surg. 2010; 145:781-4.

30. Asher KP, Gupta GN, Boris RS, Pinto PA, Linehan WM, Bratslavsky G. Robot-assisted laparoscopic partial adrenalectomy for pheochromocytoma: the National Cancer Institute technique. Eur Urol. 2011; 60:118-24. 